\title{
Estimate of Plasma Temperatures Across a CME-Driven Shock from a Comparison Between EUV and Radio Data
}

\author{
Federica Frassati $^{1}(\mathrm{D}) \cdot{\text { Salvatore } \text { Mancuso }^{1}(\mathrm{D})}$. \\ Alessandro Bemporad ${ }^{1}$
}

Received: 9 March 2020 / Accepted: 6 August 2020 / Published online: 8 September 2020

(C) The Author(s) 2020

\begin{abstract}
In this work, we analyze the evolution of an EUV wave front associated with a solar eruption that occurred on 30 October 2014, with the aim of investigating, through differential emission measure (DEM) analysis, the physical properties of the plasma compressed and heated by the accompanying shock wave. The EUV wave was observed by the Atmospheric Imaging Assembly (AIA) onboard the Solar Dynamics Observatory (SDO) and was accompanied by the detection of a metric Type II burst observed by ground-based radio spectrographs. The EUV signature of the shock wave was also detected in two of the AIA channels centered at $193 \AA$ and $211 \AA$ as an EUV intensity enhancement propagating ahead of the associated CME. The density compression ratio $X$ of the shock as inferred from the analysis of the EUV data is $X \approx 1.23$, in agreement with independent estimates obtained from the analysis of the Type II band-splitting of the radio data and inferred by adopting the upstream-downstream interpretation. By applying the Rankine-Hugoniot jump conditions under the hypothesis of a perpendicular shock, we also estimate the temperature ratio as $T_{\mathrm{D}} / T_{\mathrm{U}} \approx 1.55$ and the post-shock temperature as $T_{\mathrm{D}} \approx 2.75 \mathrm{MK}$. The modest compression ratio and temperature jump derived from the EUV analysis at the shock passage are typical of weak coronal shocks.
\end{abstract}

Keywords shock waves - Sun: activity $\cdot$ Sun: corona $\cdot$ Sun: coronal mass ejections (CMEs) - Sun: radio radiation $\cdot$ Sun: EUV radiation

\section{Introduction}

In the past decades, Type II radio bursts have been recognized as unambiguous diagnostic probes to identify the propagation of shock waves in the corona and infer their speeds (e.g. Thompson, Kennewell, and Prestage, 1996, and references therein). Both UV spectra (Raymond et al., 2000; Mancuso et al., 2002) and white-light coronagraphic observations

\footnotetext{
F. Frassati

federica.frassati@inaf.it

1 Istituto Nazionale di Astrofisica, Osservatorio Astrofisico di Torino, via Osservatorio 20, 10025 Pino Torinese, Italy
} 
(Ontiveros and Vourlidas, 2009) have been later used to yield further insight on the evolution and physical properties of coronal shock waves. More recently, EUV waves have been routinely observed as diffuse and irregular arcs of increasing coronal emission in images obtained with the EUV Imaging Telescope (EIT) onboard the Solar and Heliospheric Observatory (SOHO: Domingo, Fleck, and Poland, 1995) and with the Atmospheric Imaging Assembly (AIA: Lemen et al., 2012) onboard the Solar Dynamics Observatory (SDO: Pesnell, Thompson, and Chamberlin, 2012). However, both the physical nature of these coronal waves and their driving mechanism are not yet clear and are still under debate (e.g. Gallagher and Long, 2011; Patsourakos and Vourlidas, 2012; Warmuth, 2015). Notwithstanding the above, it is now commonly supposed that the perturbations observed in EUV coronal waves are due to fast-mode MHD waves, which, depending on the amplitude of the perturbation, can be interpreted as linear or nonlinear (shock) waves (e.g. Chen and Wu, 2011; Schrijver et al., 2011; Asai et al., 2012; Shen and Liu, 2012). In this respect, the presence of a coronal shock can be unambiguously proven by means of the simultaneous detection of Type II radio emission in the metric range. The original disturbance is often observed to be generated by an impulsive expansion of the driver (an erupting flux rope or a bubble associated with a coronal mass ejection: $\mathrm{CME}$ ) that ignites the fast-mode wave. In addition, the shock wave propagating ahead of the driver induces density enhancements due to compression and heating of the shocked coronal plasma (see Frassati et al., 2019, and the references therein). Unfortunately, there are still unsolved questions that remain to be addressed such as how and under which conditions the disturbances steepen into shock waves, how these shocks evolve, and how strong they are in terms of compression ratio and plasma heating. All these questions are also relevant in understanding where and how the solar energetic particles (SEPs), often associated with these events, are more efficiently accelerated across the shock waves.

In this article, we report the EUV analysis of an intriguing CME/shock event, occurred on 30 October 2014, with the aim to shed more light on the above questions. This event was recently analyzed in the metric radio domain by Mancuso et al. (2019) through radiospectrograph and radio-heliograph observations. The authors found that the CME-driven shock was located ahead of an expanding bubble-like EUV structure, with metric Type II radio burst emission most probably excited at the locations where the shock surface intersected the overlying streamer's axes as in the scenario proposed by Mancuso and Raymond (2004). Through 3D modeling, this analysis allowed the estimate of the density and magnetic-field strength profiles in the inner corona. In this article, taking advantage of the results obtained in Mancuso et al. (2019), we focus our attention on the detailed analysis of the EUV data retrieved by SDO/AIA to gather complementary information on the associated density compression ratio and temperature jump, thus providing a comprehensive view of this $\mathrm{CME} /$ shock event. The article is organized as follows: Section 2 is devoted to the description of the instruments and observations. Section 3 describes the method and analysis of the data. Finally, a discussion of the results and the conclusions are drawn in Section 4.

\section{Instruments and Observations}

On 30 October 2014, a solar eruption occurred near the solar limb above active region (AR)

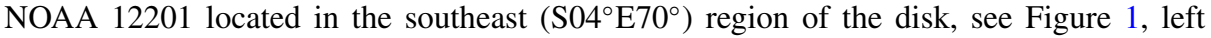
panel. This event involved a C6.9 flare observed by the GOES-15 satellite and a CME that entered the Large Angle and Spectrometric Coronagraph (SOHO/LASCO: Brueckner et al., 

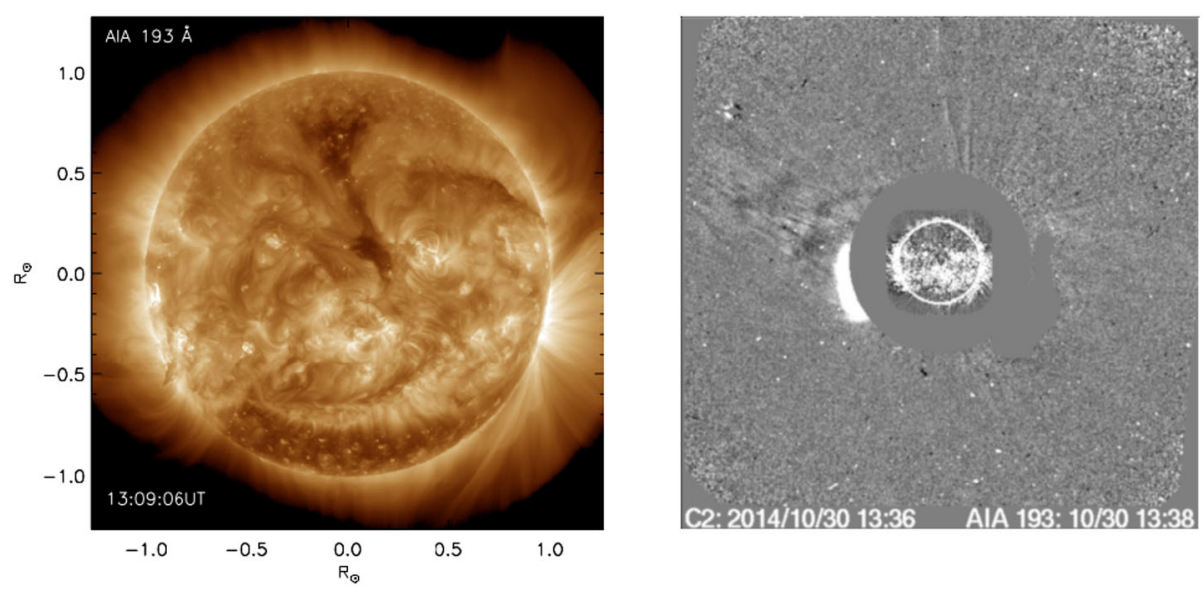

Figure 1 Left panel: SDO/AIA images of the solar corona during the event with $193 \AA$ bandpass filter. Right panel: the CME entering LASCO-C2 field of view.

1995) C2 field of view (FOV) at 13:38 UT at a height of $2.74 \mathrm{R}_{\odot}$ (Figure 1, right panel) and a mean position angle slightly greater than $90^{\circ}$ (measured counterclockwise from solar north). The CME appeared about one hour and half later in the LASCO-C3 field of view at 15:06 UT at a height of $5.44 \mathrm{R}_{\odot}$, reaching a heliocentric distance of about $15 \mathrm{R}_{\odot}$ at 21:22 UT. Unfortunately, no observations were available for this day with the Solar Terrestrial Relations Observatory (STEREO) spacecraft. The eruption propagated with a deceleration of about $-3.7 \mathrm{~m} \mathrm{~s}^{-2}$ and a projected speed, at the onset, of $\approx 350 \mathrm{~km} \mathrm{~s}^{-1}$, probably just enough to drive a weak shock. Information on the speed and deceleration of the CME was obtained from a second-order polynomial fit to the combined LASCO-C2 and -C3 heighttime measurements as derived by the Coordinated Data Analysis Workshops (CDAW) online database (cdaw.gsfc.nasa.gov).

In the inner corona, EUV data of the event have been acquired by the SDO/AIA instrument. AIA is an array of four generalized Cassegrain telescopes optimized to observe solar emissions from the transition region and corona. This instrument provides multiple simultaneous high-resolution full-disk images of the corona and transition region up to $0.5 R_{\odot}$ above the solar limb, with a field of view of at least 40 arcmin, a pixel size of 0.6 arcsec, and high temporal cadence (10-12 seconds). In case of intense events, an Automatic Exposure Control (AEC) reduces the nominal exposure time of the images in a range between 0.1 seconds and 3 seconds, depending on the intensity of the most recent image, to prevent CCD saturation. Filters on the telescopes cover ten different wavelength bands that include seven extreme ultraviolet, two ultraviolet, and one visible-light band. The seven EUV band-passes are centered on specific EUV emission lines: Fe XVIII $94 \AA(\log T \approx 6.8)$, Fe VIII-XXI $131 \AA(\log T \approx[5.6-7.0])$, Fe IX $171 \AA(\log T \approx 5.8)$, Fe XII-XXIV $193 \AA$ $(\log T \approx[6.2-7.3]), \mathrm{Fe}$ XIV $211 \AA(\log T \approx 6.3)$, He II $304 \AA(\log T \approx 4.7)$, and Fe XVI $335 \AA(\log T \approx 6.4)$. These bands were chosen to yield temperature diagnostics of the EUV emissions over the range $T \approx 0.6-20 \mathrm{MK}$.

The EUV data from SDO/AIA were used to study the EUV wave formation and evolution with time. We did not use the He II filter because this channel is more sensitive to the emission from chromospheric and transition-region plasmas. The $4096 \times 4096$ pixel (level 1) data images have undergone standard processing, which includes bad-pixel removal, despiking, and flat-fielding. 


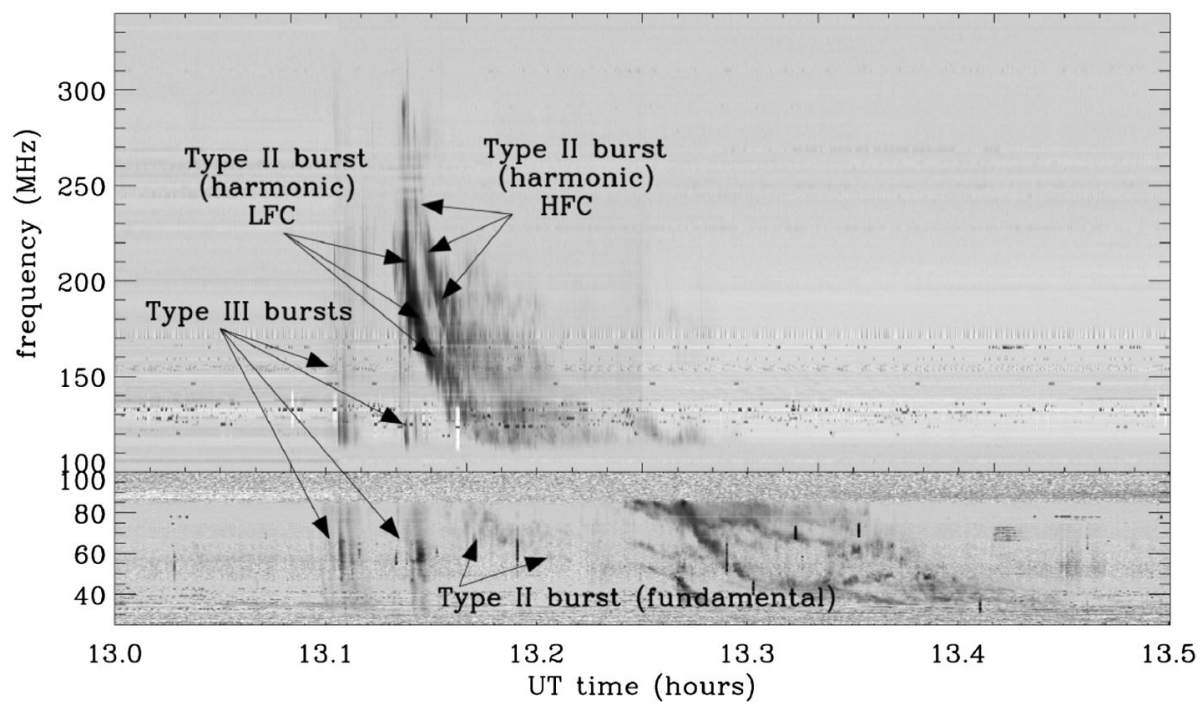

Figure 2 Combined dynamic spectra from "mid" (100 - $196 \mathrm{MHz})$ and "high" (200 - 340 MHz) BIR CALLISTO receivers covering the high-frequency range of the complex Type II/III/IV radio event detected on 30 October 2014. The "low" (25-100 MHz) range was obtained from the San Vito radio spectrometer.

For each telescope channel, the point-spread function (PSF) was deconvolved with routine aia_deconvolve_richardsonlucy.pro. All data were further processed to level 1.6 that includes a correction for plate scale, roll, and telescope coalignment, using the IDL routine aia_prep.pro. Both aforementioned routines are available within the SolarSoft (SSW) distribution. We used the NORMALIZE keyword because AIA has variable exposure times in response to the increase of EUV emission due to flare events. Further details about the AIA calibration are described by Boerner et al. (2012). To highlight the propagation of the front in EUV data, we constructed running-difference images (RDIs) by subtracting from each image of the eruption the previous one, and applying the IDL Astronomy Users Library routine filter_image.pro. This routine computes the average and/or median of the surrounding pixels (using the IDL smooth and/or median functions) in a moving box ( 3 pixels by default) replacing the center pixel with the computed average and/or median in order to enhance the fainter features. Moreover, the user can decide to deconvolve the PSF with this routine.

In the radio domain, evidence of shock-wave formation was found for this event in the data obtained from the Compound Astronomical Low-cost Low-frequency Instrument for Spectroscopy and Transportable Observatory (CALLISTO: www.e-callisto.org/) BIR spectrometer in the $200-400 \mathrm{MHz}$ frequency range and from the USAF Radio Solar Telescope Network (RSTN: ftp://ftp.ngdc.noaa.gov/STP/space-weather/solar-data/solar-features/solarradio/) spectrometer of the San Vito Observatory in the $25-180 \mathrm{MHz}$ frequency range. In general, Type II radio emissions represent unambiguous signatures of the presence of shock waves accelerating electron beams on their propagation path through the corona. The dynamic spectrum (Figure 2) of this event shows a complex Type II radio burst, mostly observed as harmonic emission, starting at about 13:08 UT and splitting into several subbands. The primary-band splitting has features suggesting shock/streamer interactions (see Mancuso et al., 2019). The further splitting of the upper-harmonic band (visible in the time interval between 13:08.5 UT and 13:08.7 UT) is most probably originating from simultaneous radio emission occurring in the upstream (ahead) and downstream (behind) region of the 

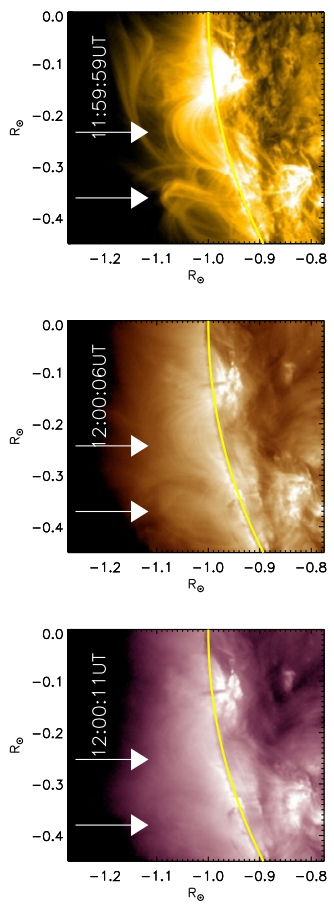
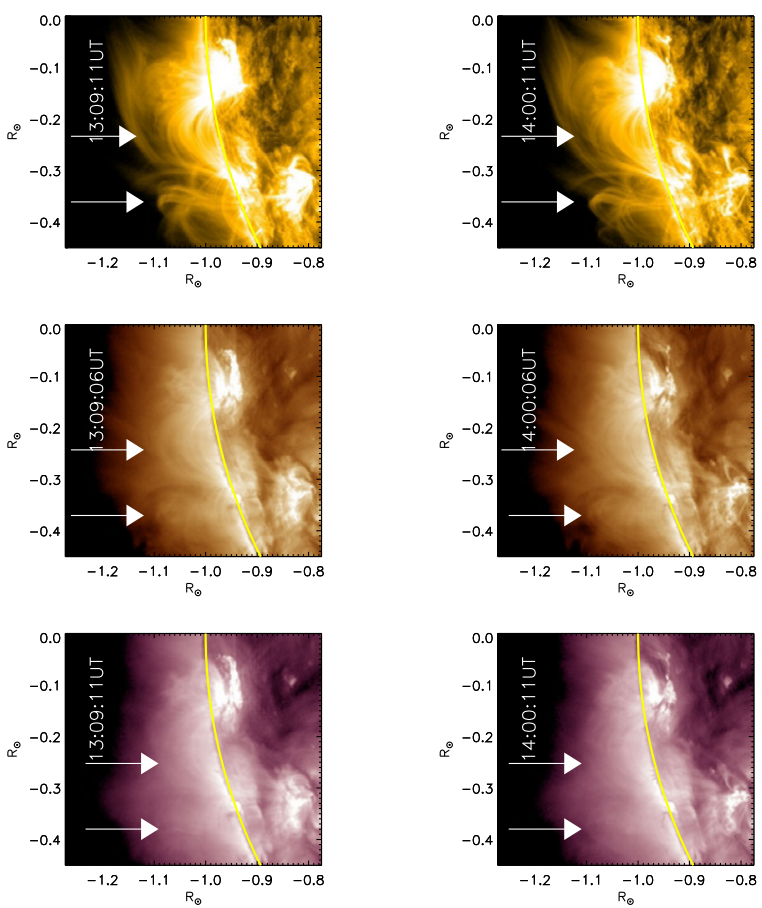

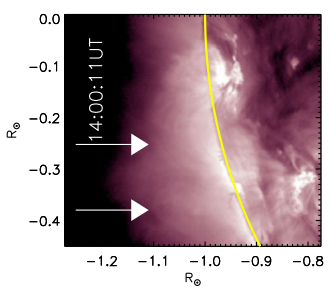

Figure 3 SDO/AIA EUV images of the solar corona in the region where the eruption occurred at $171 \AA$, $193 \AA$, and $211 \AA ̊$ before, during, and after the eruption. The coronal loops (indicated with white arrows) are well visible at $171 \AA$, less in the other filters. The yellow curve identifies the surface of the Sun.

shock front (e.g. Vršnak et al., 2001; Mancuso and Avetta, 2008; Zucca et al., 2018). The Type II radio sources were also imaged by the Nançay radioheliograph (NRH: Kerdraon and Delouis, 1997). The CME-driven shock was modeled by Mancuso et al. (2019) as a spherical expanding/translating surface assuming that the primary band-splitted Type II radio burst was emitted at the intersection of the shock surface with adjacent low-Alfvén-speed coronal streamers as in the scenario proposed by Mancuso and Raymond (2004). For further details on the instruments and the analysis of the radio data related to this event, please refer to Mancuso et al. (2019).

\section{Method and Data Analysis}

\subsection{EUV Intensity Analysis}

Figure 3 shows an enlargement of the region where the eruption occurred as observed at three different times (before, during, and after the eruption) with the $171 \AA$, $193 \AA$, and 211 $\AA$ filters. This allows one to identify the complex structure of the coronal loops (indicated with white arrows) and discriminate the quasi-stationary features from the expanding front.

Figure 4 shows SDO/AIA observations of the solar eruption and of the expanding wave front in the same EUV channels mentioned above. The acquisition times of these images are just before and during the early stages of the metric Type II radio burst. A sharp bubble-like 

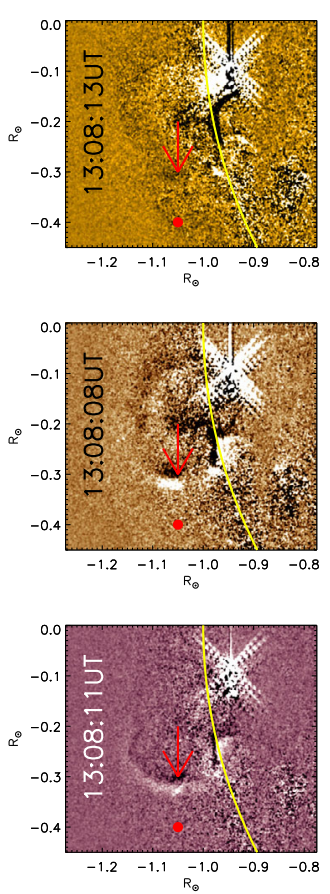
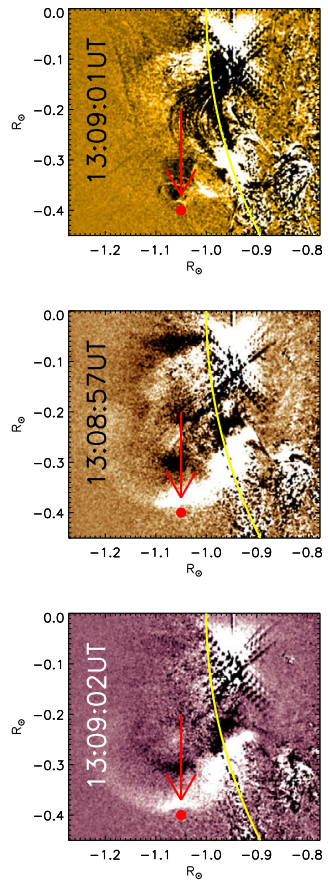
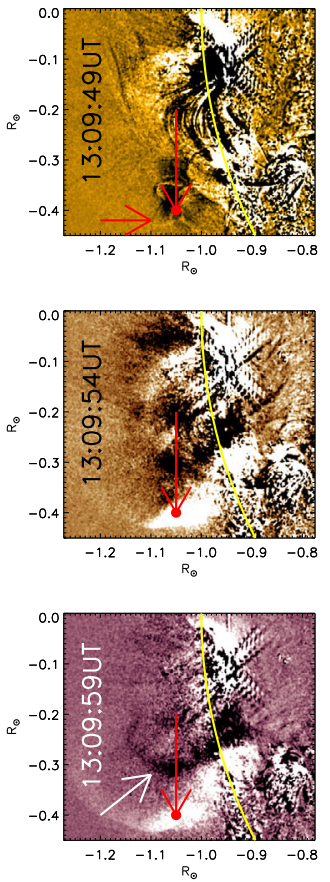

Figure 4 SDO/AIA running-difference EUV images of the eruption at $171 \AA$ (top), $193 \AA$ (middle), and 211 $\AA$ (bottom). The expanding EUV front is only visible at $193 \AA$ and appears more enhanced at $211 \AA$. The yellow curve identifies the surface of the Sun; the red dot is the area where the intensities were calculated. In all panels, red arrows indicate the expanding front. The white arrow in the image (bottom right) taken at 13:09:59 UT with the $211 \AA$ filter identifies the flux rope (also visible in the previous panel).

front expanding southward with respect to the flare location is well visible only in the $193 \AA$ and $211 \AA$ filters (see red arrows in Figure 4) although it appears fainter in the $171 \AA$ filter image. This latter feature begins to form just after 13:08 UT, around the time of the Type II radio burst onset as seen in the radio dynamic spectra (see Figure 2). Moreover, the flux rope is well visible in $211 \AA$ channel, as indicated by the white arrow in the last bottom panel. The wave lies ahead of the expanding CME bubble, as can be seen in the NRH radioheliograph observations depicted in Figure 5 (from Mancuso et al., 2019).

The characteristic peak temperatures of the observed lines (derived from temperature response function of AIA channels) suggest that the temperature of the emitting plasma in the region of interest is in the range $T \approx 1.6-4.0 \mathrm{MK}$. In order to investigate possible differences in the evolution, as observed with the EUV band-pass filters, due to temperature and/or density variations, we averaged the intensity values at different times over a fixed circular area (red dot in Figure 4) with Sun-center coordinates $\left(-1.05 \mathrm{R}_{\odot} ;-0.4 \mathrm{R}_{\odot}\right.$ ) and radius $0.01 \mathrm{R}_{\odot}$ (e.g. Ma et al., 2011; Chen, Ma, and Zhang, 2013; Vanninathan et al., 2015). This area marks the region where we measured the intensity change before and during the crossing of the shock wave and corresponds to a region in the southern part of the expanding bubble-like CME structure where the EUV front was more intense and hence more easily identifiable. Moreover, this area also corresponds to the approximate location where the harmonic component of the Type II radio burst was first imaged at high frequencies by the Nançay radioheliograph. The resulting time series of the measured intensity values, normal- 

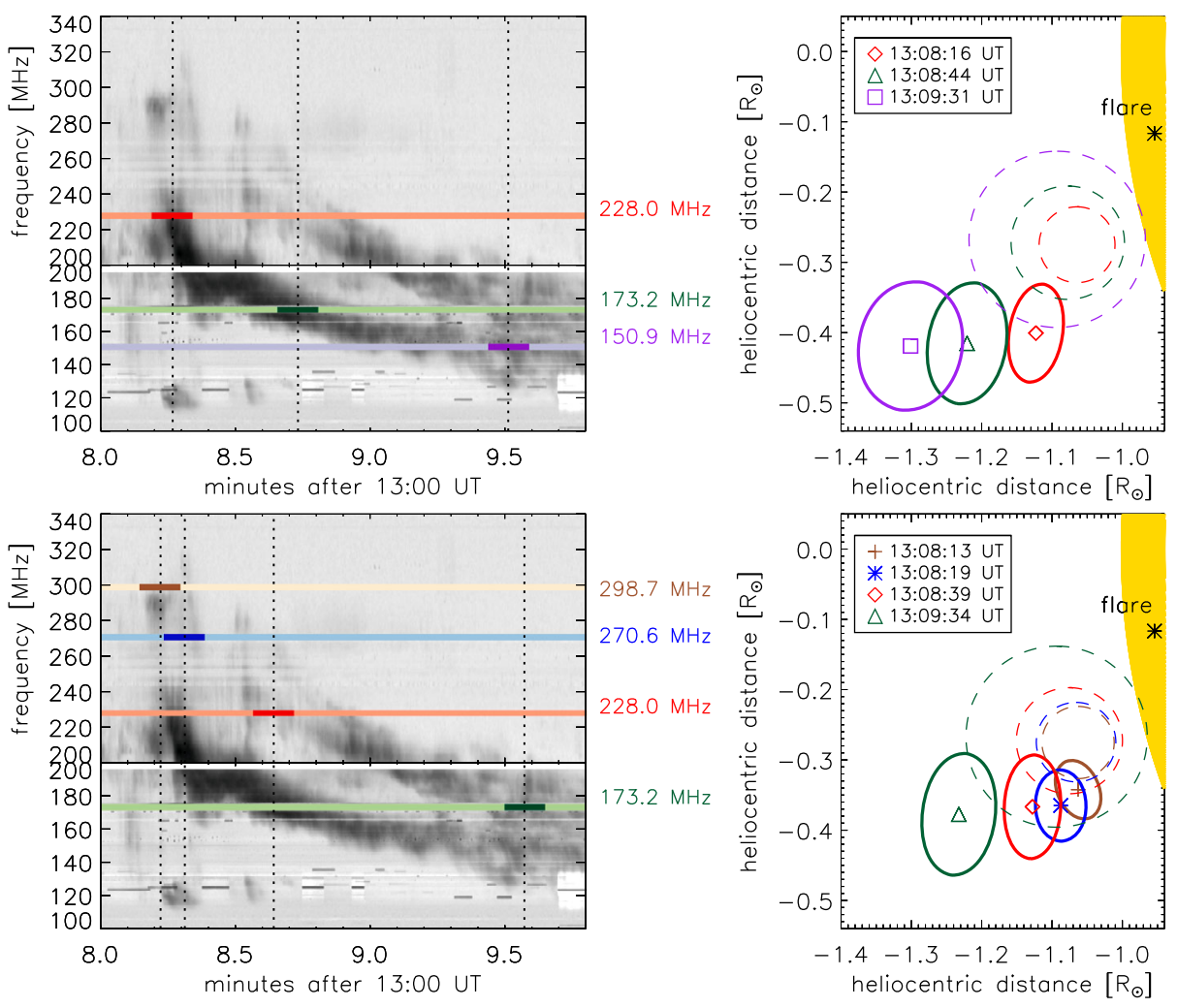

Figure 5 Left panels: Combined mid and high dynamic spectra obtained by BIR CALLISTO receivers as in Figure 2. Darker colored lines show the time intervals over which the NRH data have been averaged at a given frequency. Right panels: Locations of the centroids of 2D elliptical Gaussian fits to the Type II radio sources observed by NRH. Dashed circles correspond to the corresponding EUV front locations. Figure taken from Mancuso et al. (2019). (Reproduced with permission from Astronomy \& Astrophysics, (C) ESO)

ized to unity to allow relative comparison, are shown in Figure 6 for the $171 \AA$, $193 \AA$, and 211 A channels.

All of the EUV spectral lines included in the band-pass filters are due to collisional excitation, thus implying that their intensity is roughly proportional to the electron density squared, $n_{\mathrm{e}}^{2}$, integrated along the line-of-sight (e.g. Muhr et al., 2011; Zhukov, 2011; Goryaev et al., 2014; Del Zanna and Mason, 2018). In view of the above, if the plasma electron density $n_{\mathrm{e}}$ were the only parameter to evolve with time during the transit of the EUV front, we would expect to observe the same temporal evolution in different AIA filters. This is the case only for the evolution observed after the transit of the front around 13:10 UT, which is clearly attributable to dense CME material expanding in a thin shell ahead of the expelled flux rope. The different colored bars in Figure 6 correspond to $3 \sigma$-values above the noise level (in bold), roughly inferred by calculating the standard deviation of the intensity about six minutes before the event. The plot shows a first slow increase in the $193 \AA$ and 211 $\AA$ intensity time series at 13:08:14 UT (when the intensity variation exceeds the $3 \sigma$ level) and a corresponding slow decrease in the $171 \AA$ (first black dot-dashed line in Figure 6). This is followed by a much steeper increase in all filters around 13:08:49 UT (displayed with the second black dot-dashed line in Figure 6) that we identified as the arrival of the 

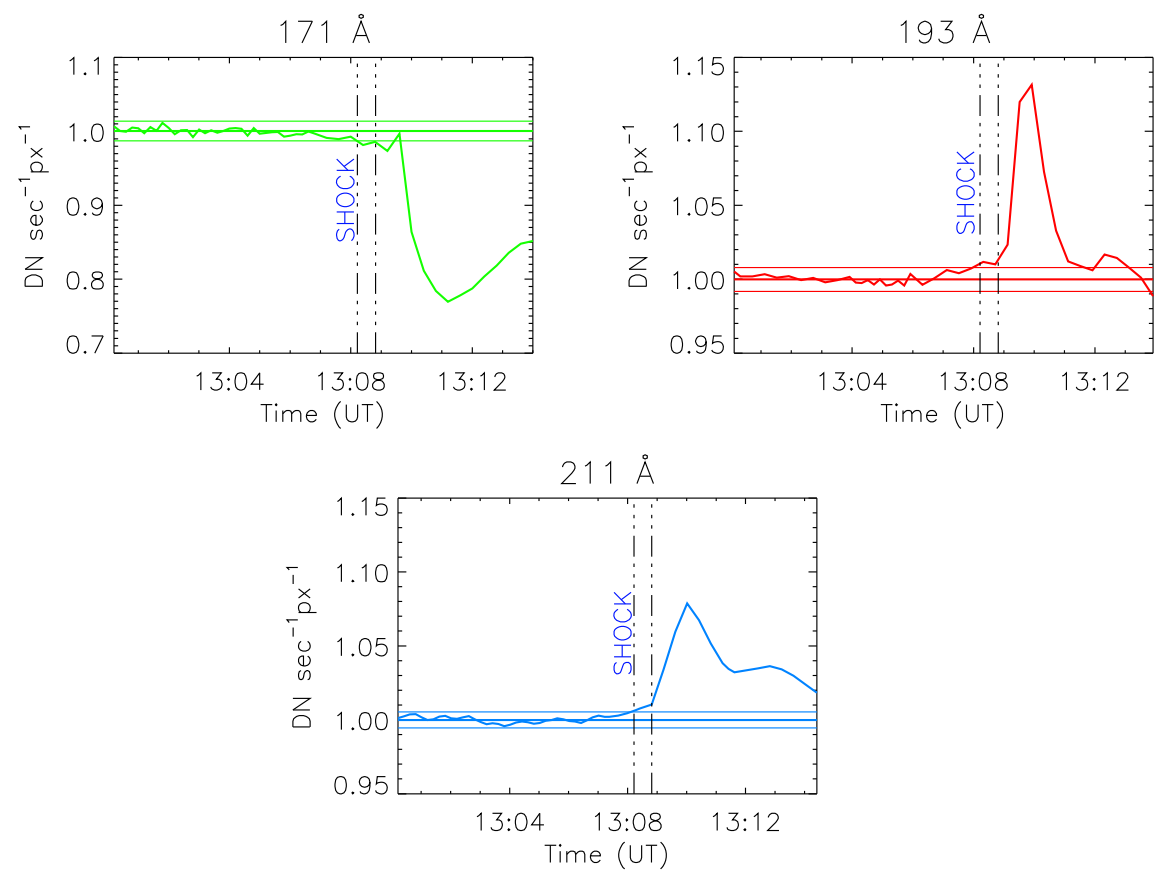

Figure 6 Intensity flux tracking of $171 \AA$, $193 \AA$, and $211 \AA$ channels in red-filled-dot region of Figure 4 . The horizontal thinner lines show the $\pm 3 \sigma$ level with respect to the average pre-event intensity. The vertical dashed lines identify the transit of the shock (13:08:14 UT) and the arrival of the flux rope (13:08:49 UT). Intensities values are normalized to unity; time starts from 13:00:00 UT.

CME flux rope. Hence, the region between the two vertical lines in Figure 6 corresponds to the shock sheath. The first slow increase is clearly attributable to the shock passage also because it is simultaneous to with the onset of the Type II radio burst and its development at high frequencies (and thus at low heliocentric distances).

After the shock passage, the $171 \AA$ intensity time series is seen to rapidly change slope, showing a little decrease before the increase, as also observed in the other two filters. The $171 \AA$ increase, due to the CME transit, appears somewhat earlier than the one observed in the $193 \AA$ and $211 \AA$ spectral lines. This effect can be attributed to a temperature gradient across the CME flux rope. After the rapid decrease, the intensities increased again after 13:12 UT, most probably due to the passage of other plasma structures in the region of interest. Together, these observations suggest that after the shock the cooler $171 \AA$ plasma was heated to 193 and $211 \AA$ A temperatures.

From the above analysis, we infer that the shock-wave front was observed in all three channels, and that it was distinctly separated from the CME bubble represented by the observed expanding EUV front. The distinctive features identifying a coronal shock with respect to other features that might be, in principle, ejected prominence or streamer material, are density compression and plasma heating. As a matter of fact, the intensities obtained from AIA imagers combine the electron density and ionization state (depending on temperature) of the plasma, thus providing a means to infer the presence of the shock from the observed images. The different slopes in the intensity fluxes clearly imply a temperature dependence. In the following section, we will show how the AIA multi-wavelength 
observations make it possible to investigate the thermal properties of the different structures observed for this event by differential emission measure (DEM) analysis.

\subsection{Parameters Derived from the DEM Method}

EUV intensity measurements are commonly reduced to study the differential emission measure, defined as the derivative of the emission measure (EM) with respect to temperature $T:$

$$
\operatorname{DEM}(T)=\frac{\mathrm{d} E M(T)}{\mathrm{d} T}=n_{\mathrm{e}} n_{\mathrm{H}}\left(\frac{\mathrm{d} T}{\mathrm{~d} s}\right)^{-1},
$$

where $n_{\mathrm{e}}$ and $n_{\mathrm{H}}$ are, respectively, the electron and hydrogen density, and $s$ is the line of sight (LOS) coordinate. The DEM is used in the local thermodynamic equilibrium approximation and estimates the emitted intensity in terms of the local temperature and gradient. Assuming that the density profile $n(s)=n_{\mathrm{e}}(s) \approx n_{\mathrm{H}}(s)$, Equation 1 can be simplified to

$$
\operatorname{DEM}(T) \sim n_{\mathrm{e}}^{2}\left(\frac{\mathrm{d} T}{\mathrm{~d} s}\right)^{-1},
$$

where $\mathrm{d} T / \mathrm{d} s$ represents the gradient of the temperature along the LOS. By using the CHIANTI code (Landi et al., 2013), an atomic database including a suite of routines to calculate the optically thin emission spectrum of EUV lines, the DEM can be fitted to the observational data in order to obtain information about the density and temperature of plasma in the emission site. The DEM-method can be applied to multi-wavelength data in order to derive temperature and EM-values (i.e. Weber et al., 2004; Cheng et al., 2012; Aschwanden et al., 2013).

In particular, Hannah and Kontar (2012) showed the capacity of regularized inversion to recover DEMs. The method uses a regularization algorithm to bind the ill-posed inverse problem: that is, DEM determination from data.

The analysis of the emission measure and the derivation of the temperature distribution in the solar corona were performed by using the intensity images at 94, 131, 171, 193, 211, and $335 \AA$ observed by SDO/AIA. In this work, we use the code to obtain 2D maps of the fundamental plasma parameters that are necessary to investigate the nature of the event. The considered plasma parameters are the emission measure $[E M]$, the temperature $[T]$, the electron density $\left[n_{\mathrm{e}}\right]$, and the plasma frequency $\left[f_{\mathrm{e}}\right]$ in the time range just before the onset and during the high-frequency evolution of the observed metric Type II radio burst.

In this analysis, the 2D DEM maps were obtained by the regularization method with zeroth-order constraint and no initial-guess solution (more detailed explanations can be found in Hannah and Kontar, 2012, 2013). To recover DEM maps, we used the IDL routine dn2dem_pos_nb.pro (www.astro.gla.ac.uk/ iain/demreg). The calculation was performed for different temperatures in the range $T_{i}=[0.5-20.0] \mathrm{MK}$ to infer the type of plasma involved in the main process.

As outlined at the beginning of the paragraph, $E M=E M(\Delta T)$ represents the amount of emitting plasma along the LOS as a function of coronal plasma temperature interval $\Delta T$ :

$$
E M(\Delta T)=\int_{\Delta T} \frac{\mathrm{d} E M(T)}{\mathrm{d} T} \mathrm{~d} T \quad\left[\mathrm{~cm}^{-5}\right] .
$$



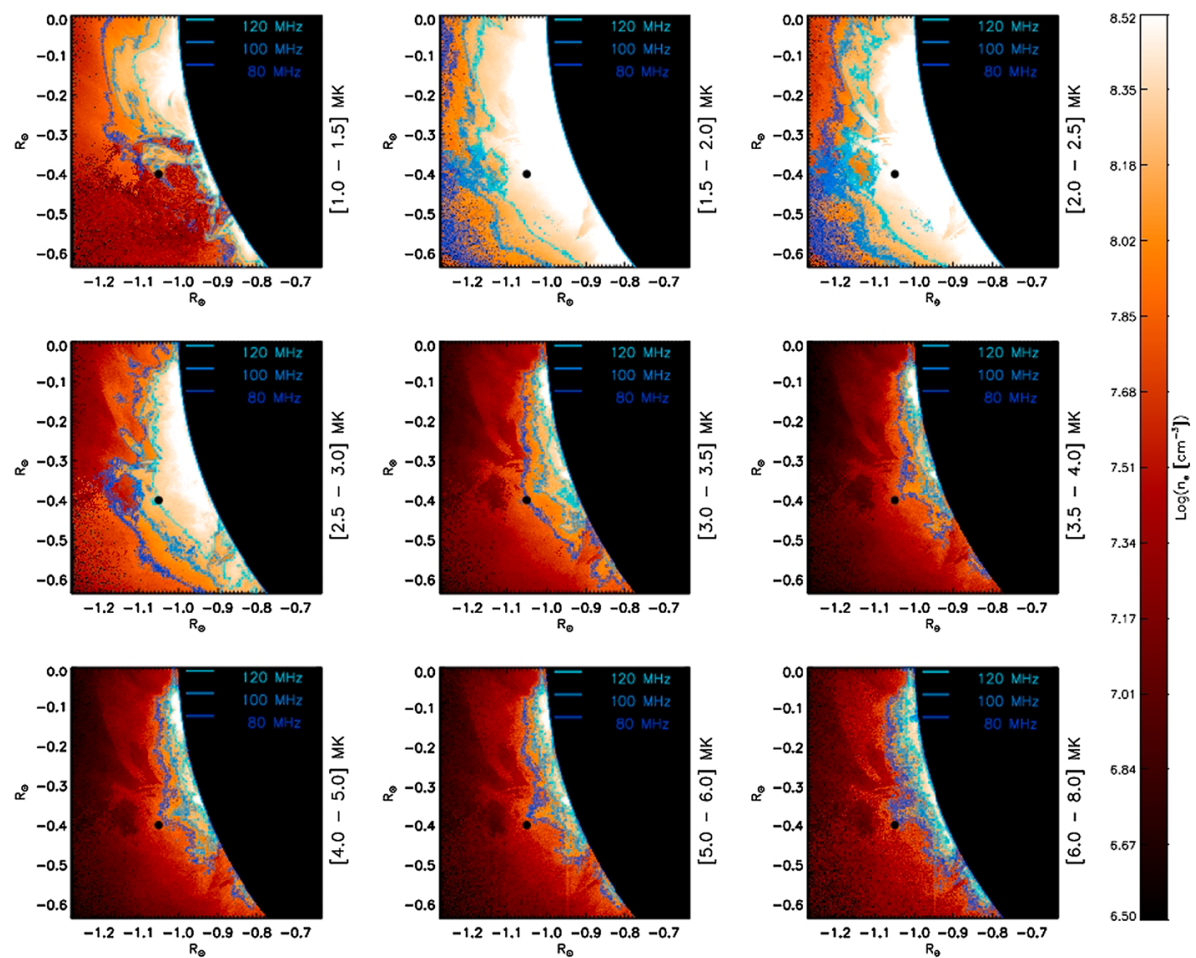

Figure 7 Electron density $n_{\mathrm{e}}$ (red-scale) 2D map and plasma frequency $f_{\text {pe }}(\mathrm{MHz}$ (blue-scale) during the initial stages of Type II radio burst (13:08:25 UT). Every map was integrated within a different temperature range, as indicated on the right of each panel. The black dot is the region where the intensities were calculated (see Section 3.1).

In order to characterize the overall temperature of the plasma, we used the DEMweighted average temperature $\langle T\rangle_{\text {DEM }}$ defined as

$$
\langle T\rangle_{\text {DEM }}=\frac{\int \mathrm{d} E M(T) T \mathrm{~d} T}{\int \mathrm{d} E M(T) \mathrm{d} T} \quad[\mathrm{~K}] .
$$

Defining the effective LOS path length $s$ as (Menzel, 1936; Zucca et al., 2014; Frassati et al., 2017)

$$
s \sim(H \pi r)^{1 / 2} \quad[\mathrm{~cm}], \quad H=\frac{\mathrm{k}_{\mathrm{B}}\langle T\rangle_{\mathrm{DEM}}}{\mu \mathrm{m}_{\mathrm{p}} \mathrm{g}} \quad[\mathrm{cm}],
$$

where $H$ is the hydrostatic scale height, $\mathrm{k}_{\mathrm{B}}$ is the Boltzmann constant, $\mu=0.61$ is the mean molecular weight, and $g$ the gravitational acceleration at the solar surface, the coronal density was calculated by using Equations 2 and 3:

$$
n_{\mathrm{e}} \approx \sqrt{\frac{E M}{s}}\left[\mathrm{~cm}^{-3}\right] .
$$

In order to make a comparison between radio and EUV observations, we compared the local plasma frequency, computed in the area where we derived the mean intensities (Figure 3), 

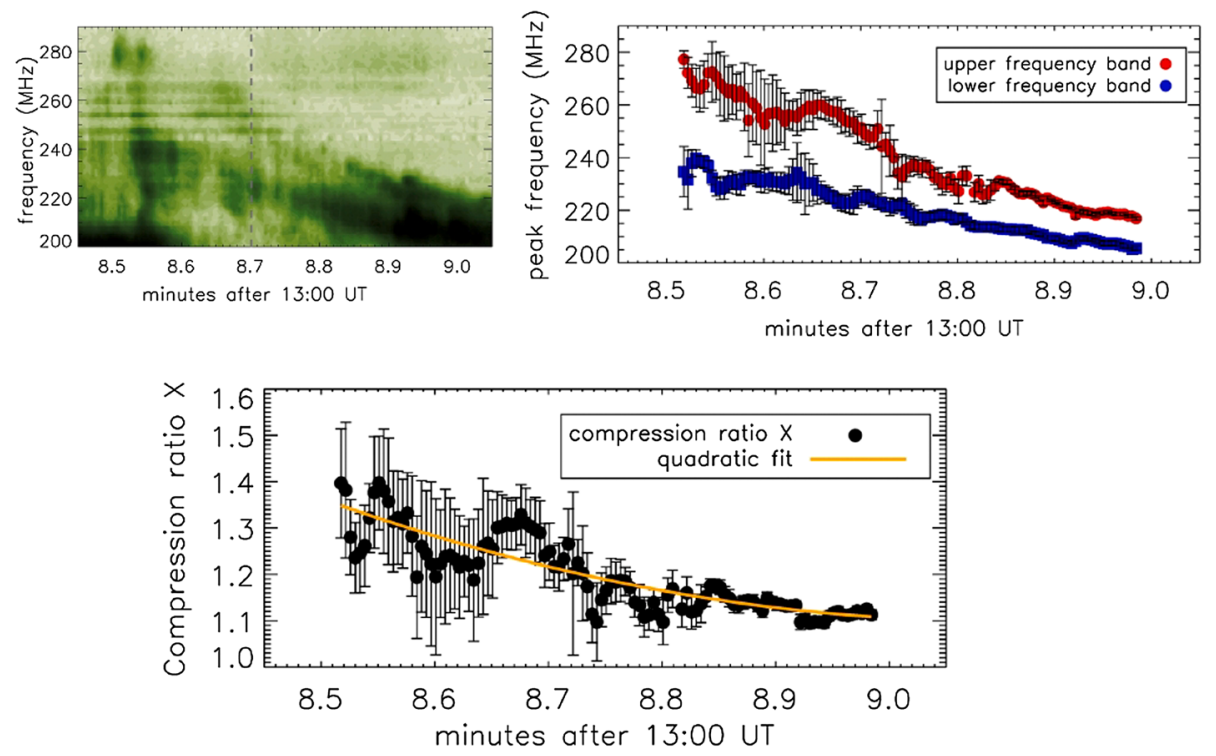

Figure 8 Top panels: Radioheliograph observations (left) and its data plots (right). Bottom panel: Compression ratio $[\mathrm{X}]$ plot derived from Equation 7.

with the starting frequency $f_{\mathrm{e}}$ of the Type II radio burst. After 13:08 UT, the Type II radio emission lanes stand out clearly, showing a weak fundamental, emitted at the electron plasma frequency $f_{\mathrm{pe}} \approx 8.98 \sqrt{n_{\mathrm{e}}\left[\mathrm{cm}^{-3}\right]} \mathrm{kHz}$ at $120 \mathrm{MHz}$ and a much stronger, splitted harmonic band at higher frequencies.

In Figure 7, we show an example of the reconstructed 2D electron-density maps at 13:08:25 UT. Superimposed on the images, we also show contour plots of the plasma frequency $\left[f_{\mathrm{pe}}\right]$ (blue-scale for each evaluated frequency) in different temperature ranges. Direct comparison between plasma frequencies $\left[f_{\mathrm{pe}}\right]$ derived from EUV at different temperature ranges, shows that only in the [2.5-3.0] MK temperature range the plasma has a frequency that is similar to the one of the Type II radio burst (the black dot lies on the $120 \mathrm{MHz}$ contour plot in all of the considered time range [13:08:00 - 13:10:00 UT]).

\subsection{Compression Ratio}

In order to study the plasma heating processes occurring at the expanding front, we investigated the compression ratio $X_{\text {EUV }}$ using the EUV data. From the combined analysis of both radio spectrometer and radioheliograph observations we have shown that the major splitting of the harmonic Type II emission seen in the dynamic spectrum of this event was due to emission from the intersection of the shock surface with two different low-Alfvénspeed coronal streamers. Here we notice that in the time interval between about 13:08.5 and 13:08.7 UT (Figure 8), the upper splitted harmonic band is further split into two subcomponents that are most probably due to simultaneous radio emission occurring in the upstream (ahead) and downstream (behind) region of a shock (e.g. Vršnak et al., 2001; Mancuso and Avetta, 2008; Zucca et al., 2018). 
This allows us to calculate the compression ratio $X_{\text {radio }}$ from the radio data as follows:

$$
X_{\text {radio }}=\frac{n_{\mathrm{e}, \mathrm{D}}}{n_{\mathrm{e}, \mathrm{U}}}=\left(\frac{f_{\mathrm{D}}}{f_{\mathrm{U}}}\right)^{2} \approx 1.1-1.4
$$

In order to infer $X_{\mathrm{EUV}}$ and make thus possible a comparison, we collected the $E M, n_{\mathrm{e}}$, $f_{\mathrm{e}}$, and $\langle T\rangle_{\mathrm{DEM}} 2 \mathrm{D}$ maps of the surrounding area where the event happened in the temporal range $\Delta t=[13: 08-13: 10] \mathrm{UT}$.

To evaluate the compression ratio across the EUV front, we need to take into account the effect of integration along the LOS. We assumed that the EUV front with thickness of $L$, transiting on the plane of the sky (POS), induces a density compression by a factor $X_{\mathrm{EUV}}$ and that $E M_{\mathrm{U}}\left(n_{\mathrm{e}, \mathrm{U}}\right)$ and $E M_{\mathrm{D}}\left(n_{\mathrm{e}, \mathrm{D}}\right)$ are the EMs up- and downstream (related with the electron densities) at times $t_{0}$ and $t_{i}$, respectively. In this analysis, $t_{0}=12: 00 \mathrm{UT}$ is the pre-event time (when the corona can be considered as stationary) and $t_{i}$ are the times at which we calculate the compression ratio in the area drawn as a black dot in Figure 7. Recalling that EM can be expressed as

$$
E M \approx \int_{\operatorname{LOS}} n_{\mathrm{e}}^{2} \mathrm{~d} L,
$$

we calculated the compression ratio $X_{\mathrm{EUV}}$ as in Frassati et al. (2019)

$$
X_{\mathrm{EUV}}=\sqrt{\frac{E M_{\mathrm{D}}-E M_{\mathrm{U}}}{P_{\mathrm{U}}}+1},
$$

where $P_{\mathrm{U}}$ is the contribution to the pre-event EM from the coronal plasma region located between $L_{1}$ and $L_{2}$ (the extension of the plasma region being compressed after the transit of the EUV front) defined as

$$
P_{\mathrm{U}}=L\left\langle n_{\mathrm{e}, \mathrm{U}}^{2}\right\rangle_{\mathrm{LOS}} \quad \text { with } \quad L=L_{2}-L_{1} .
$$

Following Bemporad and Mancuso (2010), we assumed a value of $d_{\mathrm{obs}}=0.02 R_{\odot}$ for the projected shock thickness (the diameter of the region where we performed our calculation; see the black dot in Figure 7) as observed in the SDO/AIA image. Following the results of Mancuso et al. (2019), we considered the shock surface as an expanding sphere with diameter $D=0.45 \mathrm{R}_{\odot}$ at 13:08:45 UT. These parameters were used to calculate the LOS integration length $L$ across the shock as

$$
L=\sqrt{d_{\mathrm{obs}}^{2}+2 d_{\mathrm{obs}} D} .
$$

In this calculation, we neglected the contribution of the shock motion during the exposure time. Finally, Figure 9 shows the quantity $X_{\mathrm{EUV}}$ as a function of time and temperatures. In order to make a comparison with the radio results (Figure 8), we selected a similar temporal range and, from the study performed in Section 3.1, we selected only temperature ranges between 1.5 MK and 4.0 MK. The $X_{\mathrm{EUV}}$-values are in the range of theoretical compression ratio ones: $X=[1-4]$ (using $\gamma=\frac{5}{3}$ as the adiabatic constant for a monoatomic gas).

By comparing the compression ratio calculated with radio data and those obtained for the EUV compression ratio as a function of the temperature and time, we note good agreement between the two estimates ( $X_{\mathrm{EUV}} \approx 1.15-1.23$ ) for temperatures in the range between about 2.0 and 3.0 MK. The agreement appears even better in the sub-temperature range [2.5 - 3.0] MK. 


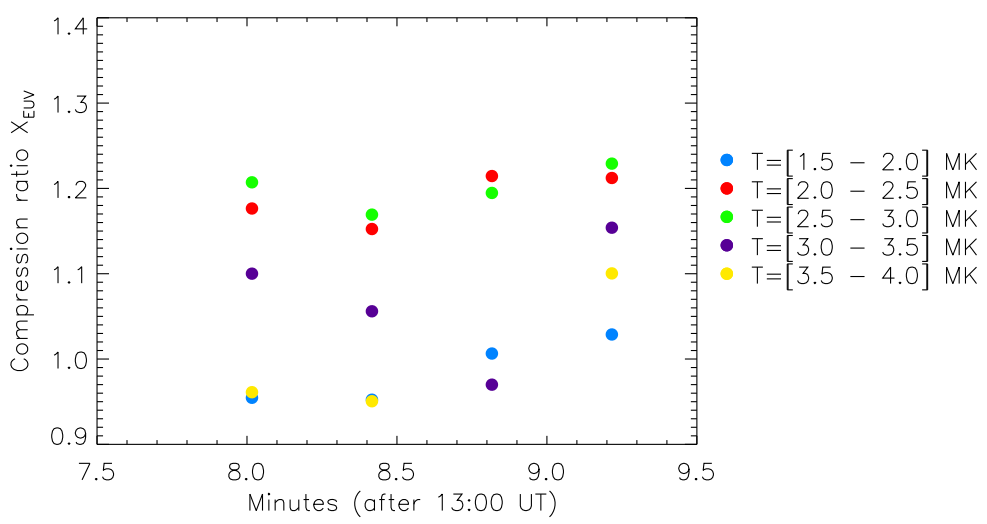

Figure 9 Temporal evolution of compression ratio in EUV wavelengths calculated for different temperature ranges.

Another method (Long et al., 2015; Zhukov, 2011; Muhr et al., 2011) that is used to calculate the compression ratio is based on the variation of the intensity observed in one single filter ( $193 \AA$ or $195 \AA$ ) and then considering only plasma with temperature in the range of the filter temperature response. This work assumed that the unknown plasma temperature changes were negligible. Moreover, since the intensity has a quadratic dependence from the density and an almost linear dependence from the temperature, they applied the following simple relationship:

$$
\frac{I_{\text {post }}}{I_{\text {pre }}} \approx \frac{n_{\mathrm{e}, \text { post }}^{2}}{n_{\mathrm{e}, \text { pre }}^{2}} .
$$

We point out that this method can be misleading because the ionization times are convolved with the timescale for the increase of emission from the shocked plasma compared to the intensity from the plasma outside the shock (foreground and background) as pointed out by Ma et al. (2011). Other authors (i.e. Kozarev et al., 2011) estimated the density jump by using the DEM and assuming that the measured intensity was emitted only from the waveaffected volume along the LOS with no change in temperature. In this case, the density ratio $n_{2} / n_{1}$ is simply given by

$$
\frac{n_{2}}{n_{1}} \sim \frac{\sqrt{\int D E M_{2}(T) \mathrm{d} T}}{\sqrt{\int D E M_{1}(T) \mathrm{d} T}} \sim \frac{\sqrt{E M_{2}}}{\sqrt{E M_{1}}} .
$$

Unfortunately, this approach can lead to an underestimate of the compression ratio.

Another important aspect to take into consideration is the temperature variation across the shock. Following Kozarev et al. (2011) and Ma et al. (2011), it is possible to obtain an estimate of the upstream temperature $T_{\mathrm{U}}$ from the total upstream- $D E M$ profile by taking the peak- $D E M$ temperature. The upstream $D E M$ curve turns out that $\log T_{\mathrm{U}}=6.25(\approx 1.78$ $\mathrm{MK})$. On the other hand, the downstream temperature cannot be derived simply from the downstream- $D E M$-peak temperature for the following reason. In this event the transit time of the shock wave in the considered regions of the corona is less than $\approx$ one minute (see time distance between the two vertical dash-dotted lines in Figure 6). This time is much shorter than the time required for ionization equilibrium for spectral lines in the considered AIA 
band-passes (see Ma et al., 2011). As a consequence, the temperature $T_{\mathrm{D}}$ cannot be estimated from the downstream- $D E M$ curves that are derived under the assumption of ionization equilibrium.

To infer the downstream temperature, it is necessary to evaluate the shock geometry. We have, however, no direct (or indirect) information about the magnetic-field direction with respect to the shock front so that, in principle, the angle between the upstream magnetic field and the normal to the shock surface $\left[\theta_{\mathrm{sh}}\right]$ could be anywhere in the range $0<\theta_{\mathrm{sh}}<\frac{\pi}{2}$. In spite of this, the condition of quasi-perpendicularity is generally supposed to be reached, as a first approximation, in the case of Type II emission in the lower corona. This is due to the fact that the acceleration mechanism of electron beams is most effective (Holman and Pesses, 1983) for this particular geometry and thus particularly favorable to the local emission of Type II radiation. Moreover, as the shock moves out from the Sun, it is easy to show that the shock geometry most probably changes from quasi-perpendicular to quasi-parallel due to the outward expansion of the shock surface, if we assume, as for this event, interaction with radial streamer structures away from the axis of symmetry of the evolving shock surface. At the very small heliocentric distance where we identified the EUV front, we thus assume, for the shock geometry, the perpendicular case. Using the results obtained by Mancuso et al. (2019) (see also the right panel in Figure 5), we can estimate the downstream temperature $T_{\mathrm{D}}$ for a perpendicular shock in the limit of $\beta \rightarrow 0$. For this calculation, we assume a sound speed $c_{\mathrm{S}}=\sqrt{\gamma \mathrm{k}_{\mathrm{B}} T_{\mathrm{U}} / \mathrm{m}_{\mathrm{H}}} \approx 165 \mathrm{~km} \mathrm{~s}^{-1}$ (where $\mathrm{k}_{\mathrm{B}}$ is the Boltzmann constant, $\gamma=\frac{5}{3}$ the adiabatic index for monoatomic gas, and $\mathrm{m}_{\mathrm{H}}$ the hydrogen mass), a shock speed $v_{\mathrm{sh}} \approx 950 \mathrm{~km} \mathrm{~s}^{-1}$, an Alfvén Mach number $M_{\mathrm{A}}=v_{\mathrm{sh}} / v_{\mathrm{A}}=1.23$, an Alfvén speed $v_{\mathrm{A}} \approx 775 \mathrm{~km} \mathrm{~s}^{-1}$, and the maximum value of the density compression ratio of $X=1.23$ in the temperature range [2.5-3.0] MK. For a perpendicular shock, the shocked plasma parameters are related to those of the unshocked plasma by the equations for conservation of momentum and energy via the Rankine-Hugoniot jump conditions. From these, the relationship between the pre-shock temperature $T_{\mathrm{U}}$ and the post-shock temperature $T_{\mathrm{D}}$ can be obtained (Priest, 1982) as

$$
\frac{T_{\mathrm{D}}}{T_{\mathrm{U}}}=\frac{1}{X}\left[1+\left(1-\frac{1}{X}-\frac{X^{2}-1}{2 M_{\mathrm{A}}^{2}}\right) \gamma M^{2}\right],
$$

where $M=v_{\mathrm{sh}} / c_{\mathrm{s}} \approx 5.76$ is the Mach number. From the right-hand side of the above equation, we infer $T_{\mathrm{D}} / T_{\mathrm{U}} \approx 1.55$, thus yielding an estimated post-shock temperature $T_{\mathrm{D}} \approx 2.75$ MK.

\section{Discussion and Conclusions}

In this article, we analyzed the formation of a CME-driven shock occurring on 30 October 2014 by using EUV data provided by the SDO/AIA instrument. Differently from other coronal instruments, the high temporal cadence of the AIA observations allows the search and spatio-temporal identification of the origin of low coronal shock waves. In fact, the intensities obtained from AIA imagers combine both electron density and ionization state (thus temperature) of the plasma, providing a powerful means to infer the presence of the shock from the observed images.

The AIA data for this event showed the appearance of an expanding EUV wave front, as classified by the Heliophysics Events Knowledgebase (HEK) system (www.lmsal.com/ isolsearch), associated with a CME bubble-like structure, starting around 13:08 UT, following a flare that occurred on the southeastern limb of the Sun. The simultaneous detection 
of a Type II radio burst in ground-based radio dynamic spectra confirmed the presence of a shock wave associated with a CME, which is supposed to be the driver of the shock. As the Type II onset is around 13:08 UT, when the EUV front is entirely in the field of view of SDO/AIA, we focused our analysis on the thermodynamic aspects of the event, and we did not study the kinematics.

By analyzing the time series of the intensity fluxes of the channels centered at $193 \AA$ and $211 \AA$, taken where the EUV front was clearly identifiable, we detected the presence of a slow increase above the $3 \sigma$-level of the unperturbed plasma preceding the CME transit that we attribute to the shock passage. In the $171 \AA$ channel, the intensity flux showed, however, a slow decrease followed by a clear, sharp intensity decrease as in the other two channels. The $171 \AA$ emission drop was interpreted as evidence of plasma heating from $171 \AA$ into the $193 \AA$ and $211 \AA$ passbands. From EM analysis of the EUV data, we were able to infer the electron density (hence, the electron plasma frequency) and the density compression ratio $X_{\mathrm{EUV}}$ for different temperature ranges. Comparison between the electron plasma frequency inferred from the EUV analysis and the one related to the Type II emission at around the same time suggests that the Type II radio burst was most likely excited in the location where the shock front was identified (around the red dot of Figure 4). From the analysis of the radio dynamic spectrum of the event, adopting the upstream-downstream interpretation for the band splitting of the upper-harmonic band, we further inferred a density compression ratio $X_{\text {radio }} \approx 1.1-1.4$. By comparing this estimate with the one obtained for $X_{\mathrm{EUV}}$ through the EUV analysis, we found a good agreement in the temperature range 2.5 3.0 MK $\left(X_{\mathrm{EUV}} \approx 1.23\right.$ in the time range 13:08:00 - 13:09:15 UT), further validating the interpretation given by Mancuso et al. (2019).

We derived the upstream temperature $\log T_{\mathrm{U}}=6.25(T \approx 1.78 \mathrm{MK})$ from the peak temperature of the differential-emission-measure profile under the hypothesis of ionization equilibrium in a stationary corona (pre-event corona). The same approach was also employed by other authors (e.g. Kozarev et al., 2011; Ma et al., 2011; Vanninathan et al., 2015) who analyzed analogous events such as the one described in this article, finding similar peak temperatures of DEM $(\log T \approx 6.17-6.25)$. These values are in agreement with the temperature response of the $193 \AA$ and $211 \AA$ filters, where the expanding front appeared as a distinct bright feature. The same hypothesis cannot be adopted to infer the downstream temperature $T_{\mathrm{D}}$ because the time required for ionization equilibrium of the spectral lines is greater than the time taken by the shock transit. Hence, assuming the presence of a perpendicular shock because of the detection of Type II radio burst and by applying the Rankine-Hugoniot jump conditions, we inferred a temperature ratio $T_{\mathrm{D}} / T_{\mathrm{U}} \approx 1.55$, thus yielding an estimated post-shock temperature $T_{\mathrm{D}} \approx 2.75 \mathrm{MK}$. The density compression ratio associated with the event analyzed in this work is small, being somewhat larger than unity and thus in the lower range of the estimates derived from white-light observations by Ontiveros and Vourlidas (2009), who found $X \approx 1.2-2.8$ for several CMEs faster than $1500 \mathrm{~km} \mathrm{~s}^{-1}$ observed by the LASCO-C2 coronagraph. The modest compression ratio characterize this event as a weak shock.

This event can be compared with the 13 June 2010 event, which has been analyzed by many authors (i.e. Kozarev et al., 2011; Ma et al., 2011; Kouloumvakos et al., 2014). The onset of the Type II associated to the event analyzed in this work appears to occur around the lateral over-expansion phase of the CME bubble, as in the 13 June 2010 event, and can play a key role in driving the shock. Moreover, the presence of complex structures, such as coronal loops and streamers, near the source region is relevant for the formation or enhancement of the shock and the excitation of Type II radio bursts. In fact, although the strength of fast-mode shocks associated with energetic CMEs is deemed to vary because of 
the uneven distribution of the ambient Alfvén speed in the coronal environment, it is very much enhanced on those parts of the wave front that encounter low- $v_{\mathrm{A}}$ structures such as streamers (see discussion by Mancuso and Raymond, 2004). Consequently, the presence of a strong Type II radio burst at the time and location in the low corona where the EUV front was identified, along with the comparable values of compression ratio derived from the analysis of both radio and EUV data, concur to indicate that the observed EUV front was a CME-driven shock wave.

Acknowledgments We thank the anonymous reviewer for very helpful and constructive comments that improved the article. Data courtesy of NASA/SDO and the AIA science team. The SOHO/LASCO data used here are produced by a consortium of the Naval Research Laboratory (USA), Max-Planck-Institut für Aeronomie (Germany), Laboratoire d'Astrophysique de Marseille (France), and the University of Birmingham (UK). SOHO is a mission of international cooperation between ESA and NASA. We are grateful to the Radio Solar Telescope Network (RSTN) and the e-CALLISTO network for providing open data access.

Funding Note Open access funding provided by Istituto Nazionale di Astrofisica within the CRUI-CARE Agreement.

Disclosure of Potential Conflicts of Interest The authors declare that they have no conflicts of interest.

Publisher's Note Springer Nature remains neutral with regard to jurisdictional claims in published maps and institutional affiliations.

Open Access This article is licensed under a Creative Commons Attribution 4.0 International License, which permits use, sharing, adaptation, distribution and reproduction in any medium or format, as long as you give appropriate credit to the original author(s) and the source, provide a link to the Creative Commons licence, and indicate if changes were made. The images or other third party material in this article are included in the article's Creative Commons licence, unless indicated otherwise in a credit line to the material. If material is not included in the article's Creative Commons licence and your intended use is not permitted by statutory regulation or exceeds the permitted use, you will need to obtain permission directly from the copyright holder. To view a copy of this licence, visit http://creativecommons.org/licenses/by/4.0/.

\section{References}

Asai, A., Ishii, T.T., Isobe, H., Kitai, R., Ichimoto, K., UeNo, S., Nagata, S., Morita, S., Nishida, K., Shiota, D., Oi, A., Akioka, M., Shibata, K.: 2012, First Simultaneous Observation of an H $\alpha$ Moreton Wave, EUV Wave, and Filament/Prominence Oscillations. Astrophys. J. Lett. 745, L18. DOI. ADS.

Aschwanden, M.J., Boerner, P., Malanushenko, A., Schrijver, C.J.: 2013, Automated Temperature and Emission Measure Analysis of Coronal Loops and Active Regions Observed with the Atmospheric Imaging Assembly on the Solar Dynamics Observatory (SDO/AIA). Solar Phys. 283, 5. ADS.

Bemporad, A., Mancuso, S.: 2010, First Complete Determination of Plasma Physical Parameters Across a Coronal Mass Ejection-driven Shock. Astrophys. J. 720, 130. DOI. ADS.

Boerner, P., Edwards, C., Lemen, J., Rausch, A., Schrijver, C., Shine, R., Shing, L., Stern, R., Tarbell, T., Title, A., Wolfson, C.J., Soufli, R., Spiller, E., Gullikson, E., McKenzie, D., Windt, D., Golub, L., Podgorski, W., Testa, P., Weber, M.: 2012, Initial Calibration of the Atmospheric Imaging Assembly (AIA) on the Solar Dynamics Observatory (SDO). Solar Phys. 275, 41. DOI. ADS.

Brueckner, G.E., Howard, R.A., Koomen, M.J., Korendyke, C.M., Michels, D.J., Moses, J.D., Socker, D.G., Dere, K.P., Lamy, P.L., Llebaria, A., Bout, M.V., Schwenn, R., Simnett, G.M., Bedford, D.K., Eyles, C.J.: 1995, The Large Angle Spectroscopic Coronagraph (LASCO). Solar Phys. 162, 357. DOI. ADS.

Chen, H., Ma, S., Zhang, J.: 2013, Overlying Extreme-ultraviolet Arcades Preventing Eruption of a Filament Observed by AIA/SDO. Astrophys. J. 778, 70. DOI. ADS.

Chen, P.F., Wu, Y.: 2011, First Evidence of Coexisting EIT Wave and Coronal Moreton Wave from SDO/AIA Observations. Astrophys. J. Lett. 732, L20. DOI. ADS.

Cheng, X., Zhang, J., Saar, S.H., Ding, M.D.: 2012, Differential Emission Measure Analysis of Multiple Structural Components of Coronal Mass Ejections in the Inner Corona. Astrophys. J. 761, 62. DOI. ADS. 
Del Zanna, G., Mason, H.E.: 2018, Solar UV and X-ray spectral diagnostics. Liv. Rev. Solar Phys. 15, 5. DOI. ADS.

Domingo, V., Fleck, B., Poland, A.I.: 1995, The SOHO Mission: an Overview. Solar Phys. 162, 1. DOI. ADS.

Frassati, F., Susino, R., Mancuso, S., Bemporad, A.: 2017, Study of the early phase of a Coronal Mass Ejection driven shock in EUV images. Astrophys. Space Sci. 362, 194. DOI. ADS.

Frassati, F., Susino, R., Mancuso, S., Bemporad, A.: 2019, Comprehensive Analysis of the Formation of a Shock Wave Associated with a Coronal Mass Ejection. Astrophys. J. 871, 212. DOI. ADS.

Gallagher, P.T., Long, D.M.: 2011, Large-scale Bright Fronts in the Solar Corona: A Review of "EIT waves". Space Sci. Rev. 158, 365. DOI. ADS.

Goryaev, F., Slemzin, V., Vainshtein, L., Williams, D.R.: 2014, Study of Extreme-ultraviolet Emission and Properties of a Coronal Streamer from PROBA2/SWAP, Hinode/EIS and Mauna Loa Mk4 Observations. Astrophys. J. 781, 100. DOI. ADS.

Hannah, I.G., Kontar, E.P.: 2012, Differential emission measures from the regularized inversion of Hinode and SDO data. Astron. Astrophys. 539, A146. DOI. ADS.

Hannah, I.G., Kontar, E.P.: 2013, Multi-thermal dynamics and energetics of a coronal mass ejection in the low solar atmosphere. Astron. Astrophys. 553, A10. DOI. ADS.

Holman, G.D., Pesses, M.E.: 1983, Solar type II radio emission and the shock drift acceleration of electrons. Astrophys. J. 267, 837. DOI. ADS.

Kerdraon, A., Delouis, J.-M.: 1997, The Nançay Radioheliograph. In: Trottet, G. (ed.) Coronal Physics from Radio and Space Observations, Lecture Notes in Physics 483, Springer, Berlin, 192. DOI. ADS.

Kouloumvakos, A., Patsourakos, S., Hillaris, A., Vourlidas, A., Preka-Papadema, P., Moussas, X., Caroubalos, C., Tsitsipis, P., Kontogeorgos, A.: 2014, CME Expansion as the Driver of Metric Type II Shock Emission as Revealed by Self-consistent Analysis of High-Cadence EUV Images and Radio Spectrograms. Solar Phys. 289, 2123. DOI. ADS.

Kozarev, K.A., Korreck, K.E., Lobzin, V.V., Weber, M.A., Schwadron, N.A.: 2011, Off-limb Solar Coronal Wavefronts from SDO/AIA Extreme-ultraviolet Observations-Implications for Particle Production. Astrophys. J. Lett. 733, L25. DOI. ADS.

Landi, E., Young, P.R., Dere, K.P., Del Zanna, G., Mason, H.E.: 2013, CHIANTI - An Atomic Database for Emission Lines. XIII. Soft X-Ray Improvements and Other Changes. Astrophys. J. 763, 86. DOI. ADS.

Lemen, J.R., Title, A.M., Akin, D.J., Boerner, P.F., Chou, C., Drake, J.F., Duncan, D.W., Edwards, C.G., Friedlaender, F.M., Heyman, G.F., Hurlburt, N.E., Katz, N.L., Kushner, G.D., Levay, M., Lindgren, R.W., Mathur, D.P., McFeaters, E.L., Mitchell, S., Rehse, R.A., Schrijver, C.J., Springer, L.A., Stern, R.A., Tarbell, T.D., Wuelser, J.-P., Wolfson, C.J., Yanari, C., Bookbinder, J.A., Cheimets, P.N., Caldwell, D., Deluca, E.E., Gates, R., Golub, L., Park, S., Podgorski, W.A., Bush, R.I., Scherrer, P.H., Gummin, M.A., Smith, P., Auker, G., Jerram, P., Pool, P., Soufli, R., Windt, D.L., Beardsley, S., Clapp, M., Lang, J., Waltham, N.: 2012, The Atmospheric Imaging Assembly (AIA) on the Solar Dynamics Observatory (SDO). Solar Phys. 275, 17. DOI. ADS.

Long, D.M., Baker, D., Williams, D.R., Carley, E.P., Gallagher, P.T., Zucca, P.: 2015, The Energetics of a Global Shock Wave in the Low Solar Corona. Astrophys. J. 799, 224. DOI.

Ma, S., Raymond, J.C., Golub, L., Lin, J., Chen, H., Grigis, P., Testa, P., Long, D.: 2011, Observations and Interpretation of a Low Coronal Shock Wave Observed in the EUV by the SDO/AIA. Astrophys. J. 738, 160. DOI. ADS.

Mancuso, S., Avetta, D.: 2008, UV and Radio Observations of the Coronal Shock Associated with the 2002 July 23 Coronal Mass Ejection Event. Astrophys. J. 677, 683. DOI. ADS.

Mancuso, S., Raymond, J.C.: 2004, Coronal transients and metric type II radio bursts. I. Effects of geometry. Astron. Astrophys. 413, 363. DOI. ADS.

Mancuso, S., Raymond, J.C., Kohl, J., Ko, Y.-K., Uzzo, M., Wu, R.: 2002, UVCS/SOHO observations of a CME-driven shock: Consequences on ion heating mechanisms behind a coronal shock. Astron. Astrophys. 383, 267. DOI. ADS.

Mancuso, S., Frassati, F., Bemporad, A., Barghini, D.: 2019, Three-dimensional reconstruction of CMEdriven shock-streamer interaction from radio and EUV observations: a different take on the diagnostics of coronal magnetic fields. Astron. Astrophys. 624, L2. DOI. ADS.

Menzel, D.H.: 1936, The Structure of the Atmosphere of $\zeta$ Aurigae, Harvard College Observatory Circular 417, HCO, Cambridge. ADS.

Muhr, N., Veronig, A.M., Kienreich, I.W., Temmer, M., Vršnak, B.: 2011, Analysis of Characteristic Parameters of Large-scale Coronal Waves Observed by the Solar-Terrestrial Relations Observatory/Extreme Ultraviolet Imager. Astrophys. J. 739, 89. DOI. ADS.

Ontiveros, V., Vourlidas, A.: 2009, Quantitative Measurements of Coronal Mass Ejection-Driven Shocks from LASCO Observations. Astrophys. J. 693, 267. DOI. ADS. 
Patsourakos, S., Vourlidas, A.: 2012, On the Nature and Genesis of EUV Waves: A Synthesis of Observations from SOHO, STEREO, SDO, and Hinode (Invited Review). Solar Phys. 281, 187. DOI. ADS.

Pesnell, W.D., Thompson, B.J., Chamberlin, P.C.: 2012, The Solar Dynamics Observatory (SDO). Solar Phys. 275, 3. DOI. ADS.

Priest, E.R.: 1982, Solar magneto-hydrodynamics, Geophys. Astrophys. Mono. 21, Reidel, Dordrecht. ADS.

Raymond, J.C., Thompson, B.J., St. Cyr, O.C., Gopalswamy, N., Kahler, S., Kaiser, M., Lara, A., Ciaravella, A., Romoli, M., O'Neal, R.: 2000, SOHO and radio observations of a CME shock wave. Geophys. Res. Lett. 27, 1439. DOI. ADS.

Schrijver, C.J., Aulanier, G., Title, A.M., Pariat, E., Delannée, C.: 2011, The 2011 February 15 X2 Flare, Ribbons, Coronal Front, and Mass Ejection: Interpreting the Three-dimensional Views from the Solar Dynamics Observatory and STEREO Guided by Magnetohydrodynamic Flux-rope Modeling. Astrophys. J. 738, 167. DOI. ADS.

Shen, Y., Liu, Y.: 2012, Simultaneous Observations of a Large-scale Wave Event in the Solar Atmosphere: From Photosphere to Corona. Astrophys. J. Lett. 752, L23. DOI. ADS.

Thompson, R.J., Kennewell, J.A., Prestage, N.P.: 1996, A Comparison of Type II Shock Speeds. Solar Phys. 166, 371. DOI. ADS.

Vanninathan, K., Veronig, A.M., Dissauer, K., Madjarska, M.S., Hannah, I.G., Kontar, E.P.: 2015, Coronal Response to an EUV Wave from DEM Analysis. Astrophys. J. 812, 173. DOI. ADS.

Vršnak, B., Aurass, H., Magdalenić, J., Gopalswamy, N.: 2001, Band-splitting of coronal and interplanetary type II bursts. I. Basic properties. Astron. Astrophys. 377, 321. DOI. ADS.

Warmuth, A.: 2015, Large-scale Globally Propagating Coronal Waves. Living Rev. Solar Phys. 12, 3 . DOI. ADS.

Weber, M.A., Deluca, E.E., Golub, L., Sette, A.L.: 2004, Temperature diagnostics with multichannel imaging telescopes. In: Stepanov, A.V., Benevolenskaya, E.E., Kosovichev, A.G. (eds.) Multi-Wavelength Investigations of Solar Activity, IAU Symp. 223, Cambridge Univ. Press, Cambridge, 321. DOI. ADS

Zhukov, A.N.: 2011, EIT wave observations and modeling in the STEREO era. J. Atmos. Solar-Terr. Phys. 73, 1096. DOI. ADS.

Zucca, P., Carley, E.P., Bloomfield, D.S., Gallagher, P.T.: 2014, The formation heights of coronal shocks from 2D density and Alfvén speed maps. Astron. Astrophys. 564, A47. ADS.

Zucca, P., Morosan, D.E., Rouillard, A.P., Fallows, R., Gallagher, P.T., Magdalenic, J., Klein, K.-L., Mann, G., Vocks, C., Carley, E.P., Bisi, M.M., Kontar, E.P., Rothkaehl, H., Dabrowski, B., Krankowski, A., Anderson, J., Asgekar, A., Bell, M.E., Bentum, M.J., Best, P., Blaauw, R., Breitling, F., Broderick, J.W., Brouw, W.N., Brüggen, M., Butcher, H.R., Ciardi, B., de Geus, E., Deller, A., Duscha, S., Eislöffel, J., Garrett, M.A., Grießmeier, J.M., Gunst, A.W., Heald, G., Hoeft, M., Hörandel, J., Iacobelli, M., Juette, E., Karastergiou, A., van Leeuwen, J., McKay-Bukowski, D., Mulder, H., Munk, H., Nelles, A., Orru, E., Paas, H., Pandey, V.N., Pekal, R., Pizzo, R., Polatidis, A.G., Reich, W., Rowlinson, A., Schwarz, D.J., Shulevski, A., Sluman, J., Smirnov, O., Sobey, C., Soida, M., Thoudam, S., Toribio, M.C., Vermeulen, R., van Weeren, R.J., Wucknitz, O., Zarka, P.: 2018, Shock location and CME 3D reconstruction of a solar type II radio burst with LOFAR. Astron. Astrophys. 615, A89. DOI. ADS. 